\title{
Multidirectional Pathways between Attachment, Mentalizing, and Posttraumatic Stress Symptomatology in the Context of Childhood Trauma
}

\author{
Yu Lien Huang ${ }^{a}$ Peter Fonagy ${ }^{b}$ Janet Feigenbaum ${ }^{b} \quad$ P. Read Montague ${ }^{c, d}$ \\ Tobias Nolte ${ }^{c, e}$ and London Personality and Mood Disorder Research \\ Consortium \\ a Department of Psychology, Fo Guang University, Yilan, Taiwan; ${ }^{b}$ Research Department of Clinical, \\ Educational and Health Psychology, UCL, London, UK; ${ }^{C}$ Wellcome Trust Centre for Neuroimaging, Institute of \\ Neurology, UCL, London, UK; ${ }^{d}$ Computational Psychiatry Unit, Virginia Tech Carilion Research Institute, \\ Roanoke, VA, USA; ${ }^{\mathrm{e}}$ Anna Freud National Centre for Children and Families, London, UK
}

\section{Keywords}

Attachment · Childhood trauma - Dissociation ·

Mentalizing $\cdot$ Posttraumatic stress disorder

\begin{abstract}
Introduction: Exposure to traumatic stressful events in childhood is an important risk factor for the development of posttraumatic symptomatology. From a mentalization-based developmental perspective, childhood adversity can affect attachment in children and may result in insecure attachment and impaired mentalizing abilities, which increase the lifetime risk for psychopathology. The present cross-sectional study examined the potential mediating role of attachment insecurity and impaired mentalizing on the relationship between childhood trauma and posttraumatic symptomatology. Method: Adults who had experienced childhood neglect and abuse ( $n=295,184$ patients with personality disorder and 111 community controls) completed self-report measures of posttraumatic stress disorder (PTSD) symptoms, dissociative experiences, adult attachment insecurity, and
\end{abstract}

\begin{tabular}{ll}
\hline KARGER & (c) 2020 The Author(s) \\
Published by S. Karger AG, Basel & This article is licensed under the Creative Commons Attribution- \\
karger@karger.com & NonCommercial-NoDerivatives 4.0 International License (CC BY- \\
NC-ND) (http://www.karger.com/Services/OpenAccessLicense). \\
Usww.karger.com/psp \\
tribution of modified material requires written permission.
\end{tabular}

mentalizing. Results: Structural equation modelling results revealed that attachment insecurity together with lower mentalizing mediated the link between childhood trauma and PTSD symptoms, and lower mentalizing mediated the link between childhood trauma and dissociative experiences. Conclusion: The findings show that attachment insecurity and lower mentalizing play significant mediating roles in the reporting of posttraumatic symptomatology among survivors of childhood abuse and neglect, with treatment implications for mentalization-based therapy as beneficial for individuals with a history of childhood trauma.

$$
\begin{aligned}
& \text { (C) } 2020 \text { The Author(s) } \\
& \text { Published by S. Karger AG, Basel }
\end{aligned}
$$

\section{Introduction}

Posttraumatic stress disorder (PTSD) is a debilitating psychological disorder that develops following traumatic life experiences, especially those occurring in childhood. From a mentalization-based developmental perspective, childhood adversity may cause insecure attachment and 
impaired mentalizing, which compromise resilience in the face of adversity throughout the life span [1]. As a result, exposure to childhood trauma may result in poorer posttraumatic adjustment than trauma experienced in adulthood $[2,3]$.

Childhood trauma is defined as all forms of physical and/or emotional ill-treatment, sexual abuse, neglect or negligent treatment, or commercial or other exploitation resulting in actual or potential harm to the child's health, survival, development, or dignity in the context of a relationship of responsibility, trust, or power; this definition includes physical abuse, psychological abuse, sexual abuse, and neglect [4]. Childhood trauma is the most common early adversity that may cause profound difficulties for posttraumatic adjustment. Adversity in early life, relative to trauma in adulthood, is more likely to disrupt the formation of secure and trusting relationships with attachment figures and the development of effective coping strategies to manage and reappraise trauma [3]. This can predispose individuals with attachment insecurity to a reduced internal feeling of security or external support in the face of later adversity and trauma, which may expose them to subsequent trauma and increase the risk for later PTSD symptoms [5]. Extensive research has demonstrated that attachment insecurity significantly predicts posttraumatic symptomatology (e.g., anxiety, depression, and PTSD symptoms) in survivors of childhood neglect and abuse $[6,7]$. In addition, the strength of the relationship between attachment insecurity and trauma symptomatology was greater for survivors of child sexual abuse than for nonabused individuals $[8,9]$. These studies indicate that attachment insecurity plays a significant role as a predictor of PTSD symptomatology after experiencing maltreatment in childhood.

Early maltreatment may not only increase the risk for the development of attachment insecurity in childhood, with long-lasting effects on attachment in intimate adult relationships, but also disrupt mentalizing abilities. Mentalizing is an ability to think about and interpret the actions of the self and others in terms of intentional mental states $[10,11]$. The development of mentalizing is thought to be fostered by secure attachment relationships. The child first learns how to mentally represent his/her own affects through the caregiver's display of the child's subjective emotional experience back to the child. In the context of a secure attachment relationship, this process of marked and contingent mirroring is believed to facilitate the development of efficient strategies to regulate distress, and to represent and communicate affects $[10,12]$.

Attachment and Mentalizing in Child Trauma
In contrast, attachment insecurity may impair the development of mentalizing [12]. Insecurely attached children, for example, those with a history of childhood abuse or neglect, have been found to have difficulties in mentalizing [13]. This is thought to be linked to the maltreating parents' compromised ability to conceive of and communicate their child's subjective emotional experience. Maltreated children then may avoid contemplating their parents' intentional mental states to reduce anxiety and to preserve their attachment relationships $[13,14]$. Through negative interactions with maltreating parents, such a child may internalize early insecure attachment relationships that, in turn, have a negative impact on the development of their mentalizing abilities. The close association between attachment insecurity and impaired mentalizing is thought to be maintained in adulthood. Insecurely attached adults are characterized by negative attachment representations regarding the perceived availability of attachment figures. Fizke et al. [15] found that the activation of insecure attachment representations significantly decreased mentalizing abilities, which manifested as compromised emotion recognition in others. Particularly, in the context of interpersonal problems or trauma, one recent study [16] found that mentalizing fully mediated the association between adult attachment insecurity and interpersonal distress, demonstrating that attachment insecurity has a negative influence on mentalizing and further contributes to interpersonal distress.

Furthermore, exposure to unresolved loss or trauma may diminish mentalizing abilities as perceiving the mental states of others as may be experienced as threatening or overwhelming [15]. This prevents traumatized individuals from effectively using social support to manage trauma and may contribute to poor posttraumatic adjustment and thus higher levels of PTSD symptomatology [12]. There is emerging evidence to indicate mentalizing failures in traumatized individuals, including lower emotional understanding [17], delayed onset of theory of mind [18], and increased difficulties in mentalizing [13, $19,20]$. Among survivors of childhood trauma, compromised mentalizing further acts as a risk factor for severe psychopathology such as personality disorders [21], as well as depressive symptoms and externalizing and sexualized behaviours $[14,22]$, indicating that mentalizing can be considered a mediator of the relationship between trauma and posttraumatic symptomatology.

Survivors of childhood trauma such as emotionally abusive parenting or child sexual abuse are also reported to experience dissociation, expressed as, for example, inhibition of negative emotional responses or expressions 
Fig. 1. The hypothesized model with the link from childhood trauma to PTSD symptoms and dissociative experiences.

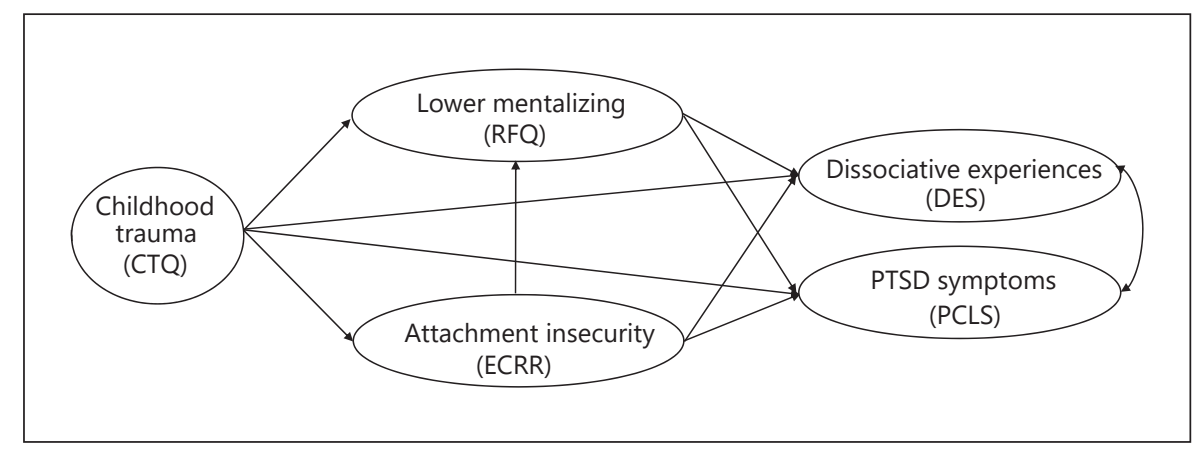

$[7,22,23]$. Childhood trauma may be viewed as a type of betrayal trauma, which refers to a betrayal of trust that is caused by individuals upon whom victims depend and who, at times, violate that trust [24]. This may contribute to a loss of epistemic trust, which describes the openness of an individual to accept new knowledge from a trustworthy person with benign intentions and whose information is relevant to the self. When traumatized individuals fail to establish epistemic trust because the intentionality of an abusive caregiver feels too dangerous to contemplate, hypervigilance with regard to learning from that caregiver and the wider social environment is paramount. As a result, reliance upon caregivers, as well as on other supportive figures via broaden-and-build experiences, as resources to cope with maltreatment is highly compromised $[25,26]$. Instead, such individuals characteristically display heightened levels of dissociation as a means to preserve the vital attachment relationship with the abusive caregiver [27]. One recent study has found that childhood sexual abuse is linked with higher levels of dissociation via lower mentalizing in the victim [22], indicating that impaired mentalizing can be considered as a potential predictor of dissociation in children who have been abused.

Given that attachment insecurity and impaired mentalizing both contribute to posttraumatic symptomatology, there is a gap in the literature insofar as no studies have addressed the role that attachment insecurity in combination with impaired mentalizing plays in the relationship between childhood trauma and posttraumatic symptomatology. The present study thus aimed to examine the effect of these two risk factors on the link between childhood trauma and PTSD symptoms and dissociation cross-sectionally in a mixed sample. We expected that attachment insecurity (here applied to current romantic partners) may have a mediating influence on impaired mentalizing. We also hypothesized that both attachment insecurity and impaired mentalizing would mediate the association between childhood trauma and PTSD symptoms and dissociation (Fig. 1).

\section{Materials and Methods}

\section{Participants}

Three hundred and forty adults (58.2\% women; age 18-65 years, mean $=30.39, \mathrm{SD}=10.08$ ) were recruited from a large-scale study in Greater London investigating personality disorders with a computational psychiatry approach. Two sample sources were used to increase sample heterogeneity. The first consisted of 221 patients who met the DSM-IV criteria for borderline personality disorder and antisocial personality disorder (58\% women, age 18 65 years, mean $=31.51, \mathrm{SD}=10.32$ ), who were referred from specialist personality disorder mental health services or a London Probation Trust-associated personality disorder screening system and had received the diagnosis of borderline and/or antisocial personality disorder based on the Structured Clinical Interview for DSM-IV axis II personality disorders [28]. Individuals with recent psychotic episodes, severe learning disabilities, or current or past neurological disorders or traumas were excluded. The second consisted of 119 community-recruited control participants $(58.4 \%$ women, age $18-52$ years, mean $=28.31, \mathrm{SD}=9.33)$. There was no sex difference between the two samples $\left(\chi^{2}(1)=0.01, p=0.945\right)$. The patient group was significantly older than the control group $(t(338)=2.82, p<0.01)$. In addition, relative to the control group, the patient group presented with higher scores on all clinical variables, including: $t(329.118)=11.62, p<0.001$ for childhood trauma; $t(334)=14.32, p<0.001$ for attachment anxiety; $t(297.495)=$ 8.94, $p<0.001$ for attachment avoidance; $t(10.441)=10.44, p<$ 0.001 for certainty about mental states; $t(19.838)=19.84, p<0.001$ for uncertainty about mental states; $t(272.615)=17.37, p<0.001$ for PTSD symptoms; and $t(313.52)=13.04, p<0.001$ for dissociative experiences.

In order to examine the influence of childhood trauma, the final sample comprised 296 participants who had experienced at least one childhood traumatic life event or experience ( $\geq$ lower severity level) screened from the Childhood Trauma Questionnaire (CTQ) [29]. There were no significant differences between those who had $(n=296)$ and had not $(n=44)$ been selected in terms of sex $\left(\chi^{2}(1)=0.02, p=0.902\right)$ and age $(t(338)=-1.76, p=0.080)$.
Huang et al. 
Participants who had experienced childhood trauma had significantly higher scores for CTQ total score $(t(328.698)=23.79, p<$ $0.01)$, attachment insecurity $(t(334)=6.86, p<0.01$ for anxiety; $t(66.348)=5.56, p<0.01$ for avoidance), PTSD symptoms $t(68.680)$ $=8.17, p<0.01)$, and dissociative experiences $t(76.559)=7.07, p<$ $0.01)$, as well as lower scores for mentalizing $(t(51.906)=-6.03$, $p<0.01$ for certainty about mental state; $t(67.527)=7.21, p<0.01$ for uncertainty about mental states).

\section{Procedure}

After providing signed informed consent, all participants were administered questionnaires for identifying childhood traumatic events and assessing the severity of posttraumatic symptoms and dissociative experiences, as well as self-reported indices of attachment. Upon completion, each participant received remuneration in cash for his/her involvement in the form of a base rate compensation of GBP 10 and additional performance-related compensation for other study components. The data set used was acquired from a wider computational psychiatry research program entitled "Probing Social Exchanges - A Computational Neuroscience Approach to the Understanding of Borderline and Anti-Social Personality Disorders" and was approved by the ethics committee of Wales.

\section{Measures}

Childhood Trauma Questionnaire. The CTQ [29] is a 28-item self-report measure on a 5 -point scale ( $1=$ never; $5=$ very often $)$. Participants respond to each item in the context of "when you were growing up." The CTQ is composed of 5 scales that assess different types of childhood trauma: emotional neglect (e.g., "felt loved"), physical neglect (e.g., "enough to eat"), emotional abuse (e.g., "someone hated you"), sexual abuse (e.g., "do sexual things"), and physical abuse (e.g., "got hit - bruises"). The questionnaire also includes a 3-item minimization/denial scale for detecting individuals who may be underreporting traumatic events. We selected participants with cut-off scores of low to moderate severity for each type of child trauma (emotional abuse $\geq 9$, physical abuse $\geq 8$, sexual abuse $\geq 6$, emotional neglect $\geq 10$, physical neglect $\geq 8$ ) [30]. The CTQ demonstrated good internal consistency in this study (Crohnbach's $\alpha=0.91,0.75,0.90,0.96$, and 0.91 for emotional neglect, physical neglect, emotional abuse, sexual abuse, and physical abuse, respectively).

Experiences in Close Relationships-Revised. The Experiences in Close Relationships-Revised (ECRR) [31] is a 36-item self-report measure that assesses adult attachment insecurity tendencies on a 7 -point scale ( 1 = strongly disagree; 7 = strongly agree) without a responding time frame. The ECRR measures 2 subscales: attachment anxiety (ECRR_AN) (i.e., the extent to which people are insecure vs. secure about the availability and responsiveness of romantic partners; e.g., "I'm afraid that I will lose my partner's love") and attachment avoidance (ECRR_AV) (i.e., the extent to which people are uncomfortable being close to and/or depending on others; e.g., "I prefer not to be too close to romantic partners"). The ECRR demonstrated good internal consistency in this study (Crohnbach's $\alpha=0.95$ and 0.94 for attachment anxiety and attachment avoidance, respectively).

Reflective Functioning Questionnaire. The Reflective Functioning Questionnaire (RFQ) [32] is a 54-item self-report measure that assesses mentalizing on a 7 -point Likert-type scale $(1=$ strongly disagree; 7 = strongly agree) without a responding time frame. The RFQ contains 2 subscales: (1) certainty about mental states
(RFQ_C; referring to the extent to which individuals disagree with statements such as "people's thoughts are a mystery to me"), which captures the respondent's level of certainty about mental states and reflects hypermentalizing with low scores; and (2) uncertainty about mental states (RFQ_U; referring to the extent to which individuals agree with an item such as "strong feelings often cloud my thinking"), which assesses the level of uncertainty about mental states, and reflects an almost complete lack of knowledge about mental states with high scores. The 8-item version of the RFQ (RFQ-8) had the highest loading on two respective factors and was therefore used in analyses. The RFQ- 8 demonstrated good internal consistency in this study (Cronbach's $\alpha=0.81$ and 0.87 for RFQ_C and RFQ_U, respectively).

Posttraumatic Stress Disorder Checklist Scale. The Posttraumatic Stress Disorder Checklist Scale (PCLS) [33] is a 17-item selfreport measured on a 5 -point Likert-type scale $(1=$ not at all; $5=$ extremely), which assesses the severity of PTSD over the past month. The PCLS comprises subscales assessing the 3 main syndromes of re-experiencing (PCLS-re; e.g., "repeated, disturbing dreams of a stressful experience from the past?"), avoidance (PCLS-avo; e.g., "feeling distant or cut off from other people?"), and hyperarousal (PCLS-aro; e.g., "being "super alert" or watchful on guard?"). The PCLS demonstrated good internal consistency in this study (Crohnbach's alphas of $\alpha=0.93,0.91$, and 0.91 for PCLSre, PCLS-avo, and PCLS-aro, respectively).

Dissociative Experiences Scale. The Dissociative Experiences Scale (DES) [34] is a 28-item self-report measure in which responses are given by circling a percentage ranging from 0 to $100 \%$ to show how often the respondent has dissociative experiences in daily life. The DES assesses dissociative experiences with regard to amnesia (DES_am; e.g., "have the experience of finding themselves in a place and having no idea how they got there"), depersonalization/derealization (DES_de; e.g., "have the experience of feeling that other people, objects, and the world around them are not real"), and absorption (DES_ab; e.g., "have the experience of being accused of lying when they do not think that they have lied"). The DES demonstrated good internal consistency in this study (Crohnbach's $\alpha=0.86$, 0.90 , and 0.87 for DES_am, DES_de, and DES_ab, respectively).

\section{Data Analysis}

The probability of missing data for all study variables was reported, and missing data were assessed by Little's missing completely at random test to examine whether data were missing at random. The regression imputation method was used to complete the data set to preserve relationships between missing values and other variables [35]. Next, an evaluation of multivariate outliers was conducted by examining the Mahalanobis distance (the distance of particular scores from the centre cluster of remaining cases) for each variable. An outlier was defined as a $\chi^{2}$ test of Mahalanobis distance greater than critical values with significance of $p<$ 0.001 . All outliers were removed from the database. Next, a test of univariate normality was performed based on skewness and kurtosis, with values of less than 2 for skewness and 7 for kurtosis [36]. Multivariate normality was tested with a critical ratio value of 1.96 for Mardia's normalized multivariate kurtosis [37].

\section{Model Specification}

The measurement model consisted of the latent variables of childhood trauma, PTSD and dissociative symptomatology, attachment insecurity, and mentalizing. The latent variable of child- 


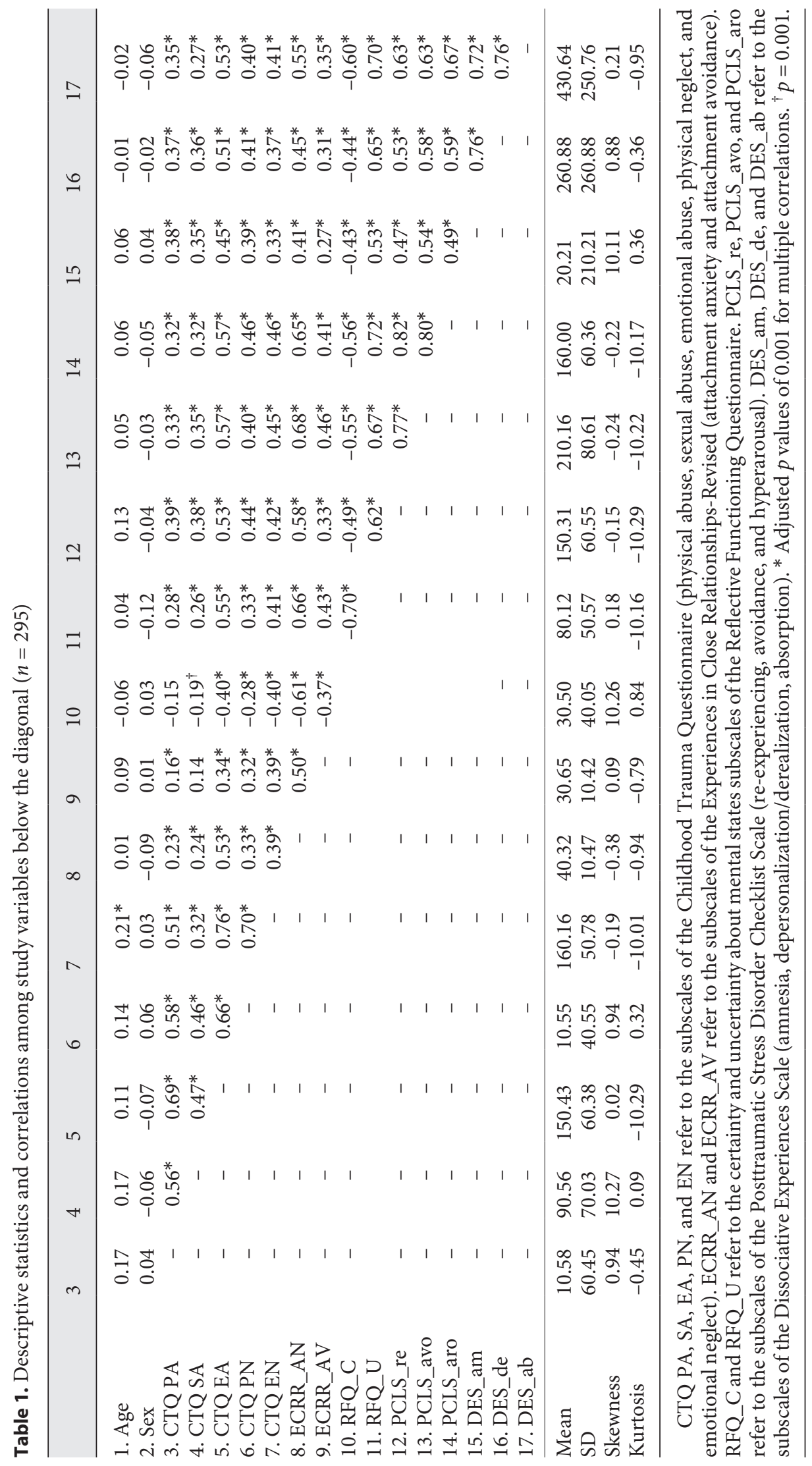


hood trauma was estimated through the following CTQ subscales: emotional neglect, physical neglect, emotional abuse, sexual abuse, and physical abuse, with higher total scores indicating greater severity of retrospectively reported childhood trauma experiences. The latent variable of PTSD symptomatology was estimated through the 3 symptoms of PTSD: re-experiencing, avoidance, and hyperarousal, with higher total scores indicating greater severity of PTSD symptoms. The latent variable dissociation symptomatology was derived from the manifest parcels amnesia, depersonalization/derealization, and absorption, with higher total scores indicating greater severity of dissociative experiences. The latent variable of attachment insecurity was assessed by 2 subscales: attachment anxiety and avoidance, with higher total scores indicating higher tendencies of attachment insecurity. The latent variable of mentalizing was also assessed by two subscales (RFQ_C and RFQ_U), with lower total scores indicating lower mentalizing. The structural model consisted of the independent variable childhood trauma and the dependent variables PTSD and dissociative symptomatology, which were mediated by lower mentalizing and attachment insecurity.

Finally, Amos 16.0 was used to test the hypothesized structural equation model (SEM) (Fig. 1). Model fits were evaluated based on indicators including: (1) a $\chi^{2}$ statistic; (2) the comparative fit index (CFI); (3) the root mean square error of approximation (RMSEA) with a $90 \%$ confidence interval (CI), with a non-significant $\chi^{2}$ statistic, CFI $\geq 0.95$, and RMSEA $\leq 0.06$ for an excellent model fit and a non-significant $\chi^{2}$ statistic, CFI $\geq 0.90$, and RMSEA $\leq 0.08$ for an adequate model fit [38]. We defined an acceptable model with the criteria of CFI $>0.90$ and RMSEA $<0.08$, and a significant $\chi^{2}$ value was expected in a large sample size.

\section{Results}

\section{Preliminary Data Analyses}

The probabilities of missing data were: age $0 \%$, sex $0 \%$, education level 1.0\%, income 5.4\%, CTQ (childhood trauma) $0 \%$, ECR-R (attachment insecurity) $0.7 \%$, RFQ (lower mentalizing) $0.3 \%$, PCLS (PTSD symptoms) $1.0 \%$, and DES (dissociative experiences) 1.0\%. The missing completely at random test showed that missing data could be assumed to be completely random $\left(\chi^{2}(159)=154.25\right.$, $p=0.592$ ). After imputation of missing data to complete the data set, the evaluation of multivariate outliers was examined and one outlier was removed, resulting in a final sample of 295 participants. The final sample consisted of 184 patients with personality disorders ( $59.2 \%$ women, with mean $=31.38, \mathrm{SD}=10.19)$ and 111 community controls $(55.9 \%$ women, age mean $=28.00, \mathrm{SD}=8.96)$. There was no difference between the groups in terms of sex $\left(\chi^{2}(1)=0.33, p=0.568\right)$, but there was a significant difference in age $(t(255.209)=2.97, p=0.003)$. The patient group was older than the control group.

The descriptive statistics for each observed variable and correlations among all study variables are presented in Table 1. For all variables, significant correlations could be established, apart from a marginally significant correlation between certainty about mental states and childhood sexual abuse, and two non-significant correlations between certainty about mental states and childhood sexual abuse, and between attachment avoidance and childhood physical abuse. In addition, there was a significant correlation between age and childhood emotional neglect, so age was controlled for in further SEM analyses.

The test of univariate normality was not violated. However, the test of multivariate normality was violated with a critical value of 2.66 for Mardia's normalized multivariate kurtosis [37], indicating a non-normal distribution. The bootstrapping maximum likelihood estimator in SEM was recommended under non-normal data condition in a large sample size [39].

\section{Structural Equation Model}

Before a structural model was tested, the measurement model was tested [40]. Confirmatory factor analysis was used to examine whether a measurement model provided an acceptable fit to the data $\left(\chi^{2}(74, n=295)=169.39, p<\right.$ 0.001 , CFI $=0.967$, RMSEA $=0.066,90 \% \mathrm{CI}=0.053-$ 0.079 ) after permitting a correlated errors term between observed variables on the same latent variable. In addition, all of the loadings of the observed variables on the latent variables and the correlations among all latent variables (i.e., CTQ, ECR-R, RFQ, DES, and PCLS) were statistically significant $(p<0.001)$.

SEM was conducted to test the hypothesized model (Fig. 1), which resulted in an adequate model fit to the data $\left(\chi^{2}(74, n=295)=169.39, p<0.001, \mathrm{CFI}=0.967\right.$, RM$\mathrm{SEA}=0.066,90 \% \mathrm{CI}=0.053-0.079)$. After removing 3 non-significant paths (childhood trauma (CTQ) on lower mentalizing (RFQ), lower mentalizing (RFQ) on posttraumatic symptoms (PCLS), and attachment insecurity (ECRR) on dissociative experiences (DES)), the adjusted model (Fig. 2) still had an adequate fit $\left(\chi^{2}(77, n=295)=\right.$ $176.84, p<0.001, \mathrm{CFI}=0.965$, $\mathrm{RMSEA}=0.066,90 \% \mathrm{CI}=$ $0.054-0.079)$. There was no significant difference between the hypothesized model and the adjusted model $\left(\chi^{2}(3)=7.45, p=0.059\right)$. Next, in order to control for potential confounders, we included age in the adjusted model, and the adjusted model did not improve $\left(\chi^{2}(87\right.$, $n=295)=205.24, p<0.001, \mathrm{CFI}=0.959$, RMSEA $=0.068$, $90 \% \mathrm{CI}=0.056-0.080$ ). The adjusted hypothesized model identified 6 significant direct paths: CTQ on ECRR, PCLS, and DES, ECR-R on RFQ and PCLS, and RFQ on DES. In addition, the model identified 4 indirect effects: 


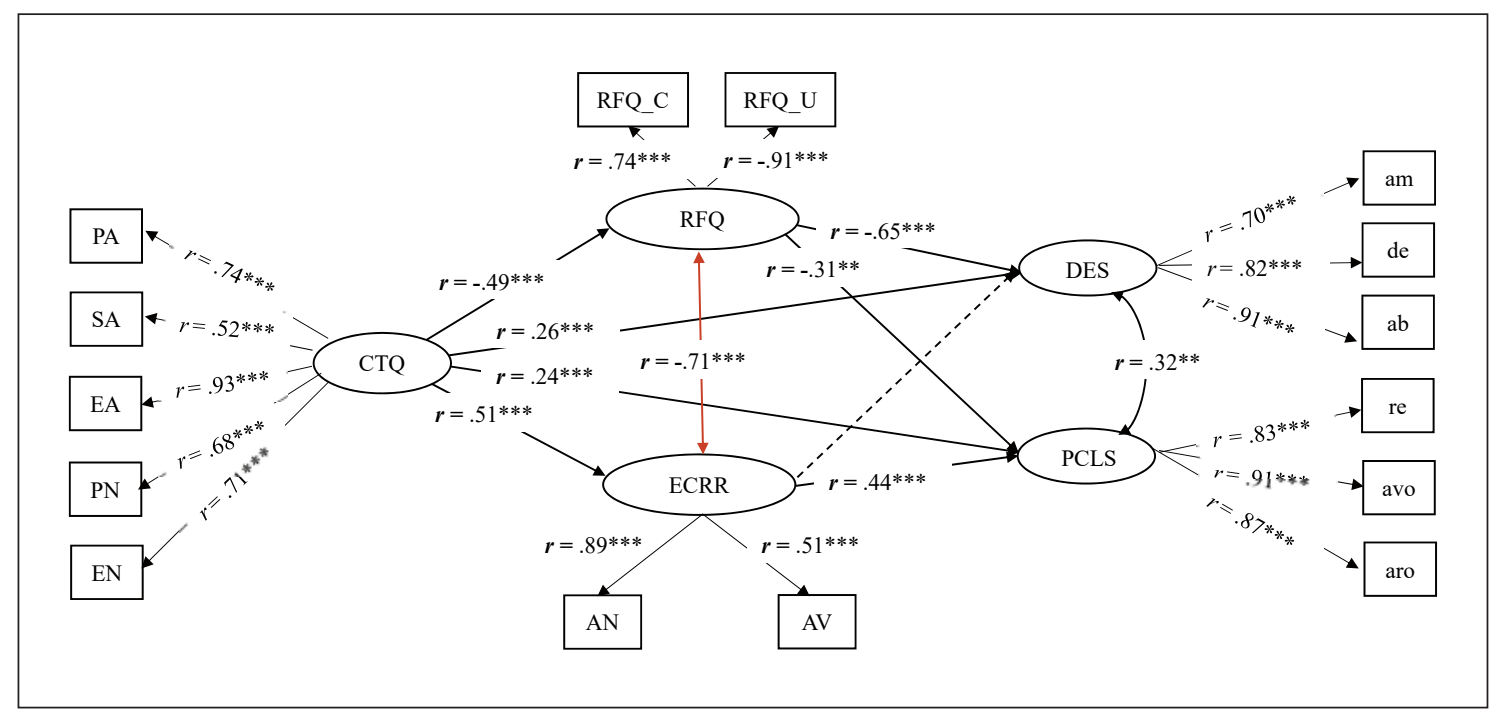

Fig. 2. The final model with attachment insecurity and lower mentalizing as mediators. ${ }^{* *} p<0.01 ;{ }^{* * *} p<0.001$.

CTQ on RFQ via ECRR, CTQ on PCLS via ECRR and RFQ, CTQ on DES via RFQ, and ECRR on DES via RFQ.

In order to rule out other plausible paths of association between attachment and mentalizing, the alternative model was tested with the order of impact from lower mentalizing to attachment. The alternative model revealed a good fit to the data $\left(\chi^{2}(74, n=295)=169.39, p<0.001, \mathrm{CFI}=\right.$ 0.967 , RMSEA $=0.066,90 \% \mathrm{CI}=0.053-0.079)$. The alternative model maintained an adequate fit after removing 1 non-significant path (ECRR on DES) $\left(\chi^{2}(75, n=295)=\right.$ $172.31, p<0.001, \mathrm{CFI}=0.966$, RMSEA $=0.066,90 \% \mathrm{CI}=$ $0.053-0.080$ ), and after adding age as a confounding variable $\left(\chi^{2}(85, n=295)=200.61, p<0.001\right.$, CFI $=0.960$, RMSEA $=0.068,90 \% \mathrm{CI}=0.056-0.080)$. The alternative model identified several significant direct (CTQ on ECRR, RFQ, PCLS, and DES; RFQ on ECR-R, PCLS, and DES; ECRR on PCLS) and indirect (CTQ on ECRR via RFQ, CTQ on PCLS via ECRR and RFQ, CTQ on DES via RFQ, and RFQ on PCLS via ECRR) paths.

The two candidate models were further examined with the Akaike information criterion (AIC), and no significant difference was noted (AIC for the adjusted hypothesized model $=335.235$, AIC for the alternative model $=$ 334.609), which justified a bidirectional arrow between ECRR and RFQ in the final model (Fig. 2). The final model resulted in an adequate fit to the data $\left(\chi^{2}(75, n=295)=\right.$ $172.31, p<0.001, \mathrm{CFI}=0.966$, $\mathrm{RMSEA}=0.066,90 \% \mathrm{CI}=$ 0.053-0.080) after removing 1 non-significant path (ECRR on DES) and adding age as a confounding variable. The final model identified several significant direct (CTQ on
Table 2. Standardized estimates and 95\% confidence intervals (CI) of each oath in structural equation modeling

\begin{tabular}{lccc}
\hline Path & $\beta$ & $95 \%$ CI & \multirow{2}{*}{$R^{2}$} \\
\hline CTQ to ECRR & $0.51^{*}$ & $0.37,0.64$ & 0.25 \\
CTQ to RFQ & $-0.49^{*}$ & $-0.58,-0.36$ & 0.23 \\
CTQ to PCLS & $0.24^{* *}$ & $0.09,0.34$ & 0.74 \\
CTQ to PCLS via ECRR and RFQ & $0.38^{* *}$ & $0.28,0.52$ & \\
ECRR to PCLS & $0.44^{*}$ & $0.15,0.79$ & \\
RFQ to PCLS & $-0.31^{\dagger}$ & $-0.55,0.02$ & \\
CTQ to DES & $0.26^{*}$ & $0.12,0.35$ & 0.66 \\
CTQ to DES via ECRR and RFQ & $0.32^{* *}$ & $0.24,0.40$ & \\
RFQ to DES & $-0.65^{* *}$ & $-0.76,-0.57$ & \\
\hline
\end{tabular}

CTQ refers to the Childhood Trauma Questionnaire. ECRR refers to the Experiences in Close Relationships-Revised. RFQ refers to the Reflective Functioning Questionnaire. PCLS refers to the Posttraumatic Stress Disorder Checklist Scale. DES refers to the Dissociative Experiences Scale. ${ }^{\dagger} p=0.062 ;{ }^{*} p<0.05 ;{ }^{* *} p<$ 0.01 .

ECRR, RFQ, PCLS, and DES; RFQ on PCLS and DES; ECRR on PCLS) and indirect (CTQ on PCLS via ECRR and RFQ, CTQ on DES via ECRR and RFQ) paths.

A bias-corrected bootstrapping procedure with $95 \%$ CIs was used to test the significance levels of indirect effects. Results showed that all the indirect effects outlined above were statistically significant (Table 2). Findings indicated that the relationship between childhood trauma and PTSD symptoms was significantly mediated by at- 
tachment insecurity together with lower mentalizing. In addition, the relationship between childhood trauma and dissociative experiences was significantly mediated by lower mentalizing related to attachment insecurity.

\section{Discussion/Conclusion}

The present study examined cross-sectional associations among adult attachment insecurity, impaired mentalizing abilities, and PTSD symptoms and dissociative experiences in the context of early maltreatment. In line with Sharp et al.'s [12] social-cognitive model of PTSD and previous empirical findings $[3,6,7,22]$, attachment insecurity together with impaired mentalizing abilities mediated associations between PTSD symptoms, dissociative experiences, and childhood neglect and abuse. While these associations cannot be interpreted in a causal way, they lend some support to a developmental mod$\mathrm{el}$, as outlined below.

We found that adult attachment insecurity together with impaired mentalizing ability mediated the link between childhood neglect and abuse and PTSD symptoms; this result indicates that attachment insecurity may be a corollary, if not a risk factor, for the development of PTSD among individuals who have experienced childhood trauma. The findings correspond to Sharp et al.'s [12] social-cognitive model of PTSD, which proposes that individuals who had early traumatic experiences with their caregivers may develop maladaptive attachment schemas regarding self and others. The activation of negative attachment schemas may distort the individual's attachment-related social information processing, as manifested by impaired mentalizing and social learning, and associated with a heightened risk of additional trauma and poorer posttraumatic adjustment (e.g., epistemic petrification, reduced use of social support).

Moreover, from the perspective of epistemic trust [11, 26], due to their maladaptive interactions with an insensitive or abusive attachment figure in childhood, insecurely attached individuals may lose trust in the information received from insensitive attachment figures, as this information may be potentially inaccurate. As a result, insecurely attached survivors of childhood trauma display epistemic hypervigilance, in which a history of attachment avoidance contributes to epistemic mistrust and an anxious attachment history leads to epistemic uncertainty regarding the validity of the information on offer. This may compromise the insecurely attached victim's ability to revise their inner knowledge (i.e., to learn from the be-

Attachment and Mentalizing in Child

Trauma nign figures in their environment) when their assumptions about themselves and their social world have been distorted by childhood trauma. Therefore, regulatory strategies required for dealing with PTSD symptomatology cannot be acquired adequately, and this places an individual at risk of PTSD following trauma.

The current study further examined the relationship between attachment and mentalizing and found it to be bidirectional. Although previous studies found that attachment insecurity contributed to mentalizing $[16,19]$, Fonagy and Bateman [41] pointed out that it would be appropriate to describe attachment and mentalizing as interrelated phenomena from different starting points in adulthood. They suggested that attachment and mentalizing may interact in a complex causal way, in which early experiences of maltreatment lead to disruptions of the attachment system, which in turn causes mentalizing failure when the attachment system is activated. This vulnerable mentalizing triggers cascades of arousal, which then undermine mentalizing even further. The potential causal association should be examined by a longitudinal study. A direct effect of childhood trauma on mentalizing was also identified in the current study. The result is in line with previous findings $[13,16-20]$ indicating that early trauma may weaken mentalizing abilities. Individuals with poorer mentalizing may perceive the mental states of others as threatening [15]. This was shown to result in a further loss of resilience in the face of trauma, which puts individuals at increased risk for PTSD following trauma [41].

Moreover, we found that lower mentalizing significantly predicted dissociation among victims of childhood abuse and neglect, consistent with the finding by Ensink et al. [22] that mentalizing difficulties increase the risk of development of dissociative experiences after exposure to childhood trauma. The combined findings confirm Sharp et al.'s [12] social-cognitive model of PTSD, which posits that representations and related mentalizing processes regarding insecure attachment experiences may link early maltreatment to later posttraumatic symptoms such as dissociative experiences. However, this may not be unique to experiences with attachment figures but may extend to extra-familial perpetrators of abuse. The CTQ, while often assumed to assess attachment trauma, also captures experiences of maltreatment such as sexual abuse by individuals outside the immediate family. Our study design cannot rule out a differential impact of these experiences.

Interestingly, attachment insecurity did not significantly predict dissociation among survivors of childhood abuse and neglect. Two types of attachment are proposed: organized attachment refers to a coherent and predictable 
pattern for caregiver-seeking when a caregiver leaves the infant in a novel environment, while disorganized attachment refers to a disruption in the coherence of the infant's strategy for seeking their caregiver (e.g., freezing or giving up in the strange situation) [42]. Particularly in the context of childhood abuse, the child perceives the caregiver as both a source of support and a threat; as a result, attachment disorganization is the major sequel of childhood trauma [43]. Childhood trauma has been proposed to engender dissociated mental representations [44], which predispose the traumatized individual to develop dissociative pathology [45]. According to Bowlby [46, 47], when individuals interact with an attachment figure accompanied with unbearable mental pain, they may distort their internal working models as a result of dissociation between conscious and unconscious working models to minimize anxiety. Liotti [45] further suggested that disaggregated representations of the self and the caregiver are a typical characteristic of disorganized attachment, and dissociative symptoms may emerge as a consequence of activation of the disorganized attachment system in the face of trauma. In light of the above, disorganized attachment, but not organized forms of insecure attachment (i.e., anxiety and avoidance), may better explain the relationship between childhood trauma and dissociation. This may underpin the fact that, in the present study, we did not find a significant mediating role of attachment insecurity - as an organized strategy - for the association between childhood trauma and dissociation.

\section{Limitations}

Several limitations of the present study warrant consideration. First, the study did not assess different attachment representations categorically and thus could not explore the impact of disorganized attachment on dissociation. A measure assessing individual differences in attachment (e.g., the Adult Attachment Interview) should be used in future studies to replicate our findings. Second, no information was gathered regarding the time interval between the occurrence of trauma and participation in the study, due to the limitation of the instruments we used to assess trauma. Hence, in future studies a measure of trauma that includes an assessment of the specific time interval since trauma occurred should be used. Third, there was collinearity between PTSD symptoms and dissociation. A dissociation subtype of PTSD has been proposed in the DSM-5 [48]. PTSD symptoms may co-occur with dissociation and required the inclusion of a correlation between two variables in SEM analysis. This restricted us from elucidating the impact of attachment and mentalizing on the PTSD symptoms and dissociation separately. Fourth, and most importantly, our study employed a cross-sectional design and was based on self-reported indices of the relevant constructs of interest; this design was unable to verify the potentially causal relationships among study variables. Further research within a longitudinal design is needed to determine the causal relationship between either parent-infant or adult romantic attachment insecurity, childhood trauma, mentalizing, and trauma-related symptoms.

\section{Clinical Implications}

Given that the development of mentalizing abilities promotes resilience in the face of adversity, mentalization-based treatment $[49,50]$, which is designed to foster mentalizing abilities and transference-focused psychotherapy [51], which has been shown to improve attachment status and reflective function, as well as other evidence-based approaches, would likely be beneficial for individuals with a history of childhood trauma. Furthermore, a secure attached therapeutic relationship is helpful to form epistemic trust, a protective factor enhancing resilience to adversity through higher-order cognitive processes of perspective-taking and flexible reappraisal of past experiences [25, 41, 52].

\section{Acknowledgement}

The authors gratefully acknowledge funding by the Wellcome Trust (Read Montague) under a Principal Research Fellowship, and the Kane Foundation (Read Montague). Tobias Nolte is supported by the Wellcome Trust Principal Research Fellowship Award to Prof. Read Montague. Peter Fonagy is in receipt of a National Institute for Health Research Senior Investigator Award (NF-SI-0514-10157). Peter Fonagy was in part supported by the National Institute for Health Research (NIHR) Collaboration for Leadership in Applied Health Research and Care (CLAHRC) North Thames at Barts Health NHS Trust. Yu Lien Huang was in receipt of the Ministry of Science and Technology of Taiwan for a Postdoctoral Research Fellowship Award (104-2917-I564-003-A1). The views expressed are those of the author(s) and not necessarily those of the NHS, the NIHR or the Department of Health. The authors would like to thank all the research assistants and students who helped with data collection.

\section{Statement of Ethics}

The study was approved by the ethics commission of Wales. All participants completed an informed consent form that included the purpose and the procedures of the study, any potential consequences of participating in the study, as well as information regarding the right to decline to participate in the study.
56

Psychopathology 2020;53:48-58 DOI: $10.1159 / 000506406$
Huang et al. 


\section{Disclosure Statement}

The authors declare no conflicts of interest.

\section{Author Contributions}

Dr. Fonagy, Dr. Montague, and Dr. Nolte developed the theoretical formalism. Dr. Feigenbaum and Dr. Huang contributed to sample preparation. Dr. Huang performed the analytic calculation. Both Dr. Huang and Dr. Nolte contributed to the final version of the paper. Dr. Fonagy and Dr. Montague supervised the project. All authors provided critical feedback and helped shape the research and manuscript.

\section{References}

1 Fonagy P, Luyten P. A developmental, mentalization-based approach to the understanding and treatment of borderline personality disorder. Dev Psychopathol. 2009;21(4): 1355-81.

2 Chu DA, Williams LM, Harris AW, Bryant RA, Gatt JM. Early life trauma predicts selfreported levels of depressive and anxiety symptoms in nonclinical community adults: relative contributions of early life stressor types and adult trauma exposure. J Psychiatr Res. 2013 Jan;47(1):23-32.

3 Ogle CM, Rubin DC, Siegler IC. The relation between insecure attachment and posttraumatic stress: early life versus adulthood traumas. Psychol Trauma. 2015 Jul;7(4):324-32.

4 Butchart A, Kahane T, Phinney HA, Mian M, Furniss T. Preventing child trauma: a guide to taking action and generating evidence. Geneva: WHO and International Society for the Prevention of Child Abuse and Neglect; 2006. pp. 7-10.

5 Mikulincer M, Shaver PR, Solomon Z. An attachment perspective on traumatic and posttraumatic reactions. In: Safir MP, Wallach HS, Rizzo AS, editors. Future directions in post-traumatic stress disorder. New York (NY): Springer; 2015. pp. 79-96.

6 Bifulco A, Kwon J, Jacobs C, Moran PM, Bunn A, Beer N. Adult attachment style as mediator between childhood neglect/abuse and adult depression and anxiety. Soc Psychiatry Psychiatr Epidemiol. 2006 Oct;41(10): 796-805.

7 Twaite JA, Rodriguez-Srednicki O. Childhood sexual and physical abuse and adult vulnerability to PTSD: the mediating effects of attachment and dissociation. J Child Sex Abuse. 2004;13(1):17-38.

8 Aspelmeier JE, Elliott AN, Smith CH. Childhood sexual abuse, attachment, and trauma symptoms in college females: the moderating role of attachment. Child Abuse Negl. 2007 May;31(5):549-66.

9 Whiffen VE, Judd ME, Aube JA. Intimate relationships moderate the association between childhood sexual abuse and depression. J Interpers Violence. 1999;14(9):940-54.

10 Fonagy P, Gergely G, Jurist EL, Target M. Affect regulation, mentalization, and the development of the self. New York (NY): Other Press; 2002.
11 Fonagy P, Luyten P, Allison E, Campbell C. Reconciling psychoanalytic ideas with attachment theory. In: Cassidy J, Shaver PR, editors. Handbook of attachment: theory, research, and clinical applications. New York (NY): Guilford Press; 2016. pp. 780-804.

12 Sharp C, Fonagy P, Allen J. Posttraumatic stress disorder: social-cognitive perspective. Clin Psychol. 2013;19:229-40.

13 Ensink K, Normandin L, Target M, Fonagy P, Sabourin S, Berthelot N. Mentalization in children and mothers in the context of trauma: An initial study of the validity of the Child Reflective Functioning Scale. Br J Dev Psychol. 2015 Jun;33(2):203-17.

14 Ensink K, Bégin M, Normandin L, Fonagy P. Maternal and child reflective functioning in the context of child sexual abuse: pathways to depression and externalising difficulties. Eur J Psychotraumatol. 2016 Jan;7(1):30611.

15 Fizke E, Buchheim A, Juen F. Activation of the attachment system and mentalization in depressive and healthy individuals: an experimental control study. Psihologija (Beogr). 2013;46(2):161-76.

16 Hayden MC, Müllauer PK, Gaugeler R, Senft B, Andreas S. Mentalization as mediator between adult attachment and interpersonal distress. Psychopathology. 2019;52(1):10-7.

17 Shipman K, Zeman J, Penza S, Champion K. Emotion management skills in sexually maltreated and nonmaltreated girls: a developmental psychopathology perspective. Dey Psychopathol. 2000;12(1):47-62.

18 O'Reilly J, Peterson CC. Maltreatment and advanced theory of mind development in school-aged children. J Fam Violence. 2015; 30(1):93-102.

19 Brüne M, Walden S, Edel MA, Dimaggio G. Mentalization of complex emotions in borderline personality disorder: the impact of parenting and exposure to trauma on the performance in a novel cartoon-based task. Compr Psychiatry. 2016 Jan;64:29-37.

20 Petersen R, Brakoulias V, Langdon R. An experimental investigation of mentalization ability in borderline personality disorder. Compr Psychiatry. 2016 Jan;64:12-21.

21 Chiesa M, Fonagy P. Reflective function as a mediator between childhood adversity, personality disorder and symptom distress. Pers Ment Health. 2014 Feb;8(1):52-66.
22 Ensink K, Bégin M, Normandin L, Godbout $\mathrm{N}$, Fonagy P. Mentalization and dissociation in the context of trauma: implications for child psychopathology. J Trauma Dissociation. 2017 Jan-Feb;18(1):11-30.

23 Collin-Vézina D, Hébert M. Comparing dissociation and PTSD in sexually abused school-aged girls. J Nerv Ment Dis. 2005 Jan; 193(1):47-52.

24 Freyd JJ. Betrayal trauma: traumatic amnesia as an adaptive response to childhood abuse. Ethics Behav. 1994;4(4):307-29.

25 Fonagy P, Allison E. The role of mentalizing and epistemic trust in the therapeutic relationship. Psychotherapy (Chic). 2014 Sep; 51(3):372-80.

26 Nolte T, Campbell C, Fonagy P. Social Communicative Processes in Severe Personality Disorder. In: Bizzari V, Gonçalves J, Pereira JG, editors. The psychotherapy-neurobiology-pharmacology intervention triangle. Wilmington (DE): Vernon Press; 2018. pp. 161-84.

27 Hulette AC, Kaehler LA, Freyd JJ. Intergenerational associations between trauma and dissociation. J Fam Violence. 2011;26(3):21725.

28 First MB, Gibbon M, Spitzer RL, Williams JB, Benjamin LS. Structured Clinical Interview for DSM-IV Axis II Personality Disorders, (SCID-II). Washington (DC): American Psychiatric Press; 1997.

29 Bernstein DP, Fink L. Childhood Trauma Questionnaire: a retrospective self-report manual. San Antonio (TX): Psychological Corporation; 1998.

30 Bernstein DP, Stein JA, Newcomb MD, Walker E, Pogge D, Ahluvalia T, et al. Development and validation of a brief screening version of the Childhood Trauma Questionnaire. Child Abuse Negl. 2003 Feb;27(2):169-90.

31 Fraley RC, Waller NG, Brennan KA. An item response theory analysis of self-report measures of adult attachment. J Pers Soc Psychol. 2000 Feb;78(2):350-65.

32 Fonagy $\mathrm{P}$, Luyten $\mathrm{P}$, Moulton-Perkins A, Lee YW, Warren F, Howard S, et al. Development and validation of a self-report measure of mentalizing: the Reflective Functioning Questionnaire. PLoS One. 2016 Jul;11(7): e0158678. 
33 Weathers F, Litz B, Herman D, Huska J, Keane T. The PTSD Checklist (PCL): reliability, validity, and diagnostic utility. Paper presented at the Annual Convention of the International Society for Traumatic Stress Studies, San Antonio (TX). Oct 1993.

34 Bernstein EM, Putnam FW. Development, reliability, and validity of a dissociation scale. J Nerv Ment Dis. 1986 Dec;174(12):727-35.

35 Zhang Z. Missing data imputation: focusing on single imputation. Ann Transl Med. 2016 Jan;4(1):9.

36 West SG, Finch JF, Curran PJ. Structural equation models with nonnormal variables: problems and remedies. In: Hoyle RH, editor. Structural equation modeling: concepts, issues, and applications. Thousand Oaks (CA): Sage Publications; 1995. pp. 56-75.

37 Mardia KV. Measures of multivariate skewness and kurtosis with applications. Biometrika. 1970;57(3):519-30.

38 Hu LT, Bentler PM. Cutoff criteria for fit indexes in covariance structure analysis: conventional criteria versus new alternatives. Struct Equ Modeling. 1999;6(1):1-55.

39 Nevitt J, Hancock GR. Performance of bootstrapping approaches to model test statistics and parameter standard error estimation in structural equation modeling. Struct Equ Modeling. 2001;8(3):353-77.
40 Anderson JC, Gerbing DW. Structural equation modeling in practice: a review and recommended two-step approach. Psychol Bull. 1988;103(3):411-23.

41 Fonagy P, Bateman AW. Adversity, attachment, and mentalizing. Compr Psychiatry. 2016 Jan;64:59-66.

42 Main M, Solomon J. Procedures for identifying infants as disorganized/disoriented during the Ainsworth Strange Situation. In: Greenberg MT, Cicchetti D, Cummings EM, editors. Attachment in the preschool years: Theory, research, and intervention. London: The Unviersity of Chicago Press; 1990. pp. 121-60.

43 van Ijzendoorn $\mathrm{MH}$, Schuengel C, Bakermans-Kranenburg MJ. Disorganized attachment in early childhood: meta-analysis of precursors, concomitants, and sequelae. Dev Psychopathol. 1999;11(2):225-49.

44 Riggs SA. Childhood emotional abuse and the attachment system across the life cycle: what theory and research tell us. J Aggress Maltreat Trauma. 2010;19(1):5-51.

45 Liotti G. A model of dissociation based on attachment theory and research. J Trauma Dissociation. 2006;7(4):55-73.
46 Bowlby J. Separation. Attachment and loss. Volume 2. New York (NY): Basic Books; 1973.

47 Bowlby J. Loss. Attachment and loss. Volume 3. New York (NY): Basic Books; 1980.

48 American Psychiatric Association. Diagnostic and statistical manual of mental disorders (DSM-5). Washington (DC): American Psychiatric Pub; 2013.

49 Allen JG. Mentalizing in the development and treatment of attachment trauma. London: Karnac Books; 2013.

50 Allen JG. Coping with trauma: Hope through understanding. 2nd ed. Washington (DC): American Psychiatric Publishing; 2008.

51 Kernberg OF, Diamond D, Yeomans FE, Clarkin JF, Levy KN. Mentalization and attachment in borderline patients in transference focused psychotherapy. In: Jurist EL, Slade A, Bergner S, editors. Mind to mind: Infant research, neuroscience, and psychoanalysis. New York (NY): Other Press; 2008. pp. 167-201.

52 Debbané M, Nolte T. Contemporary neuroscientific research. In: Bateman A, Fonagy P, editors. Handbook of mentalizing in mental health practice. Washington (DC): American Psychiatric Association Publishing; 2019. pp. $21-36$. 